\title{
A Comparison of Didodecyldimethylammonium Bromide Adsorbed at Mica/Water and Silica/Water Interfaces using Neutron Reflection
}

\author{
Lucy R. Griffin ${ }^{\mathrm{a}}$, Kathryn L. Browning ${ }^{\mathrm{a}}$, Chris L. Truscott ${ }^{\mathrm{a}}$, Luke A. Clifton ${ }^{\mathrm{b}}$, John Webster ${ }^{\mathrm{b}}$ and \\ Stuart M. Clarke ${ }^{\mathrm{a} *}$ \\ ${ }^{a}$ BP Institute, University of Cambridge, Madingley Road, Cambridge, Cambs, CB3 0EZ, UK \\ ${ }^{\mathrm{b}}$ ISIS, Rutherford Appleton Laboratory, Didcot, Oxfordshire, UK \\ * Corresponding author email: stuart@bpi.cam.ac.uk, Tel: +00 44 (0) 1223 765700, Fax: +00 44 (0) 1223 \\ 765701
}

\begin{abstract}
The layer structure of the dichain alkyl ammonium surfactant, didodecyldimethylammonium bromide (DDAB), adsorbed from water on to silica and mica surfaces has been determined using neutron reflection. Although sometimes considered interchangeable surfaces for study, we present evidence of significant differences in the adsorbed layer structure below the critical micelle concentration.

A complete DDAB bilayer was assembled at the water/mica interface at concentrations below the critical micelle concentration (CMC). In contrast it is not until the CMC was reached that the complete bilayer structure formed on the oxidised silicon crystal. Removal of the complete bilayer on both surfaces was attempted by both washing and ion exchange yet the adsorbed structure proved tenacious.
\end{abstract}

\section{Key Words}

Mica, Silica, Neutron Reflection, DDAB, Didodecyldimethylammonium Bromide, Surfactant, Adsorption

\section{Introduction}

The neutron reflection (NR) technique is a powerful tool for structure determination at interfaces. Its capability for "contrast variation" by isotopic H/D substitution of both the adsorbate and solvent allows extra information to be obtained from systems which are essentially chemically identical but scatter neutrons differently. Each component of the system has a scattering length density from the sum of all the isotopes within the material. This helps to remove the somewhat ambiguous nature of structure determination. However, the technique is not without its difficulties; for optimised experiments samples should be extremely flat with low roughness over a large area, allowing a sufficient reflected signal to be 
collected in a reasonable time. These restrictions can limit the number of different mineral surfaces it is possible to study. Silicon/silica, quartz and sapphire crystals have all been extensively studied due the availability of large defectless single crystals and their low attenuation cross sections ${ }^{1-9}$.

Information from NR studies often supplements that from other surface specific techniques such as surface force apparatus (SFA) and atomic force microscopy (AFM). For these techniques, muscovite mica is often the substrate of choice due to the exceptional flatness achieved by cleaving the mica layers, negating the need for extensive polishing (as with silicon/sapphire/quartz). These techniques require the introduction of a second surface or probe onto the adsorbate during data collection. It has been shown that this can introduce deformation in delicate surface structures and induce crystallisation in specific systems ${ }^{10,11}$. In contrast, neutron reflection is a non-invasive probe of interfacial structures.

In order to perform complementary measurements using all these methods, it would be convenient to experimentally study adsorption to mica using NR. However, single crystals of mica large enough for reflectivity studies (greater than $25 \times 25 \mathrm{~mm}$ ) are very uncommon. Crystals of this size tend to include defects which cause additional scattering of the beam which, coupled with a large attenuation cross section, mean penetration through bulk mica is problematic.

Attempts have been made previously by Cosgrove et al but experienced a number of experimental difficulties ${ }^{12}$. As a result of these difficulties, comparison is often drawn between adsorbate structures imaged using AFM/SFA on mica and those determined using neutron reflection technique on other surfaces $^{13-15}$. These comparisons rely on the assumption that adsorbate structures are not significantly affected by the differences between mica and other substrates. It is likely that there will be rather different behaviour in the adsorption on mica from other silicates. The silica surface consists of $\mathrm{OH}$ groups that can be protonated/deprotonated leading to a strongly $\mathrm{pH}$ dependent surface charge in direct contrast to the $\mathrm{pH}$ independent structural charge of mica, resulting from isomorphic substitution ${ }^{16}$.

Recently it has been shown that the difficulties accompanying neutron reflection from bulk mica can be circumvented by supporting a thin layer of mica on a silicon substrate ${ }^{17,18}$. As a result, we can directly compare adsorbate structures at the mica/water and silicon/water interfaces using neutron reflection, and here present evidence indicating adsorption on silica and mica is not the same.

Here we present a detailed study of the adsorption and desorption behaviour of DDAB at both the mica/water and silica/water interfaces. The surfactant adsorption isotherm up to the CMC was determined for each surface, highlighting interesting differences in the adsorption behaviour of the surfactant on the two surfaces. Reversibility of the adsorption through both washing and ion exchange with monovalent and divalent salt species was also investigated 


\section{The Silica Surface}

Crystalline silica is composed of a combination of Si-O bonds which include Si-O-Si bridging oxygens and hydrated Si-OH groups. Each oxygen atom may have one or two associated protons as shown by Iler $^{19}$. In solution the charge of the silica surface is governed by the protonation and deprotonation of these $\mathrm{Si}-\mathrm{OH}$ and $\mathrm{Si}-\mathrm{OH}_{2}$ groups. These equilibria are $\mathrm{pH}$ sensitive and, as such, the surface charge is also a function of $\mathrm{pH}$. The isoelectric point of silica is rather low at approximately $\mathrm{pH} 2^{19}$. Hence on raising the $\mathrm{pH}$, deprotonation of $\mathrm{Si}-\mathrm{OH}$ groups at the surfaces results in a negative charge. This negative surface increases most strongly up to approximately $\mathrm{pH} 6$ where after it increases much more slowly. The negative charge at the silica surface must be compensated by counterions which can be protons or other ions in solution including inorganic cations or cationic surfactants.

\section{The Mica Surface}

Muscovite mica is a dioctahedral phyllosilicate mineral with unit formula $\mathrm{KAl}_{2}\left(\mathrm{AlSi}_{3} \mathrm{O}_{10}\right)(\mathrm{F}, \mathrm{OH})_{2}$. The bulk structure is comprised of repeating layers with each layer containing an octahedral $\left(\mathrm{Al}_{2} \mathrm{O}_{3}\right)$ sheet sandwiched between two tetrahedral $\left(\mathrm{SiO}_{4}\right)$ sheets. In muscovite mica, approximately a quarter of the silicon atoms in the tetrahedral sheet are isomorphically substituted for aluminium resulting in a single negative charge for each $47 \AA^{2}$ of the surface $e^{20,21}$. This high degree of substitution in mica means that the surface has a high charge density compared to other clay minerals and leads to a zeta potential of up to $150 \mathrm{mV}$ in distilled water ${ }^{22,23}$. The surface charge remains constant with varying $\mathrm{pH}$ owing to the structural nature of the charge. In the naturally occurring mineral, potassium ions compensate the layer charge and sit on the basal plane between the layers. These bound cations act to pin adjacent layers to one another, this strong interaction makes mica a non-swelling clay (unlike montmorillonite for instance). Muscovite mica is easily cleaved uniformly over large areas between the covalently bonded layers, exposing the basal plane which consists of a hexagonal array of oxygen atoms/potassium ions. During the cleavage process half the potassium ions remain associated with each layer; these surface ions may be exchanged with other ions including surfactants when exposed to an aqueous solution. Hence, we expect the mica will behave differently from silica reflection substrates where the surface charge originates from protonation or deprotonate of surface and is a strong function of $\mathrm{pH}$.

\section{DDAB Surfactant}




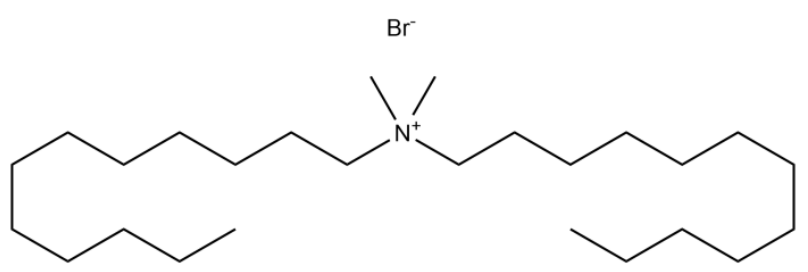

Figure 1: Illustration of the molecular structure of didodecyl dimethyl ammonium bromide (DDAB)

\begin{tabular}{cc}
\hline Parameter & Value \\
\hline $\mathrm{l}_{\mathrm{c}}{ }^{\mathrm{a}} / \AA$ & 16.68 \\
$\mathrm{~A}_{0}{ }^{\mathrm{b}} / \AA^{2}$ & 68 \\
$\mathrm{~V}_{\mathrm{c}}{ }^{\mathrm{c}} / \AA^{3}$ & 703.7 \\
$\mathrm{P}$ & 0.620
\end{tabular}

Table 1: Molecular dimensions and packing parameters for surfactants used. ${ }^{a}$ Alkyl chain length obtained using Tanford's formula $l_{c}=1.50+1.265$ (no of carbons). ${ }^{b}$ Head group area, $A_{0}$, calculated from aggregation numbers of singlechain surfactants assuming spherical micelles. 'Surfactant chain volume obtained using Gruen's equation, $\mathrm{V}_{\mathrm{c}}=\mathbf{5 4 . 3 0 ( n o}$ of $\left.\mathrm{CH}_{3}\right)+27.05$ (no of $\mathrm{CH}_{2}$ ). Packing parameter, $\mathrm{P}$, is given by $\mathrm{Eq} 1$.

A first estimate of the shape/geometry of a given surfactant aggregate can be given from the packing parameter $(P)$ :

$P=\frac{\mathrm{V}_{\mathrm{c}}}{\mathrm{A}_{0} \mathrm{l}_{c}}$

Where $V_{c}$ is surfactant chain volume, $A_{0}$ the head group area and $l_{c}$ the alkyl chain length ${ }^{24}$.

Didodecyldimethyl ammonium bromide has a packing parameter of 0.620 and so is expected to form flat (either lamellae or vesicle) structures at concentrations above the CMC. This has been shown by small angle $\mathrm{x}$-ray scattering ${ }^{25}$. Similarly one would expect the formation of flat, bilayer structures above the $\mathrm{CMC}$ at the solid/liquid interfaces.

The adsorption behaviour of this surfactant, DDAB, on mica has been reported using $\mathrm{AFM}^{13}$. Uniform and featureless images were interpreted to indicate a flat bilayer formed at the surface at $0.30 \mathrm{mM}$ (above the $\mathrm{CMC}$ ). In a recent publication by our group, DDAB was studied at the mica/water interface with neutron reflection ${ }^{18}$. It was found that a $24 \AA$ bilayer which contained almost no water was formed at the surface at twice the CMC; in good agreement with the results from the AFM study.

Schultz et al and Blom et al made similar observations about the adsorption of DDAB above the CMC on quartz comparing both AFM images and NR data ${ }^{1,6}$. The bilayer thickness reported by both authors was in the range 23 - $26 \AA$; a thickness consistent with the value determined on mica and the thickness of lamellae in free solution ${ }^{25}$. 
The effect of washing with water and $\mathrm{KCl}$ solutions on the $\mathrm{DDAB}$ bilayer was also investigated in our recent paper ${ }^{18}$. Interestingly the adsorbed layer was found to be very tenacious. It was found that after copious washing with pure water only $15 \%$ of the bilayer was removed. Even after 1 hour of rinsing with $1 \mathrm{M} \mathrm{KCl}$ solution only $43 \%$ of the surfactant was removed. The remainder of the surfactant could only be removed by UV ozone treatment of the surface which removed the organic layer by chemical reaction.

\section{Materials and Methods}

\section{Samples}

The didodecyldimethylammonium bromide (Sigma Aldrich certificate of analysis $100.1 \%$ by argentometric titration with an IR spectra that conforms to the molecule expected) was additionally checked for purity using drop shape analysis, elemental analysis, ${ }^{1} \mathrm{H}$ and ${ }^{13} \mathrm{C}$ NMR, and LC-MS. No minimum in the surface tension as a function of solution concentration was detected prior to the CMC, indicating the absence of surface active impurities at the air/liquid interface. The literature reports a range of values for the CMC of didodecyldimethylammonium bromide (DDAB) between 0.05 and 0.15 $\mathrm{mM}^{1,13,26-28}$. We determined the CMC as $0.08 \mathrm{mM}$ and this is the value referred to throughout this study. The other material characterisation techniques conformed to expectations for the DDAB molecule and indicated that the sample contained no significant contamination could be identified. If there is a small amount of impurity, this is expected to be the monochain amine that will not be preferentially adsorbed over the dichain of interest here.

The $\mathrm{D}_{2} \mathrm{O}$ used in this study was provided by ISIS and the $\mathrm{H}_{2} \mathrm{O}$ used was from an ultrapure source (Millipore $18.2 \mathrm{M} \Omega \cdot \mathrm{cm}$ ). Contrast changes were made using an HPLC pump (L7100 HPLC pump, Merck, Hitachi), a total of $24 \mathrm{~mL}$ (14 cell volumes) at $2 \mathrm{~mL} \mathrm{~min}^{-1}$ was passed through the sample cell for each solution exchange to ensure the change of sample was as complete as reasonably possible. Sample cells were designed specifically to ensure good liquid exchange properties. Waters described as contrast matched to silicon have a scattering length density comparable to silicon $\left(2.07 \times 10^{-6} \AA^{-2}\right)$. Solutions of DDAB in $\mathrm{D}_{2} \mathrm{O}$ and $\mathrm{H}_{2} \mathrm{O}$ were prepared at $2 \mathrm{CMC}(0.16 \mathrm{mM})$. Surfactant solutions $(0.1,0.5$ and $1 \mathrm{CMC})$ were prepared by mixing the required ratios of $\mathrm{D}_{2} \mathrm{O}, \mathrm{H}_{2} \mathrm{O}, 2 \mathrm{CMC}$ DDAB in both $\mathrm{D}_{2} \mathrm{O}$ and $\mathrm{H}_{2} \mathrm{O}$ in the HPLC pump before passage to the sample cell. All surfaces were allowed to equilibrate with the solutions for a short time during reflectometer alignment procedures before measurement; additional equilibration time was not considered necessary due to the immediate observation of the lamella phase. 


\section{Sample Preparation}

Silicon substrates for reflection were prepared as follows: $100 \mathrm{~mm} \times 50 \mathrm{~mm} \times 10 \mathrm{~mm}$ silicon wafers (Crystran n-type material (111) face) were cleaned in concentrated nitric acid for four hours to remove organic contaminates, washed ten times in ultra-pure water and allowed to soak overnight. Crystals were removed from the water and placed in a UV Ozone cleaner (Bioforce Nano) for 15 minutes to remove any remaining contaminates just before use. The crystal was visually inspected for scratching or damage and run under ultra-pure water to check complete hydrophilicity of the surface. Once complete, the crystal was clamped against a nitric cleaned Teflon trough between two metal plates to make a solid/liquid interface.

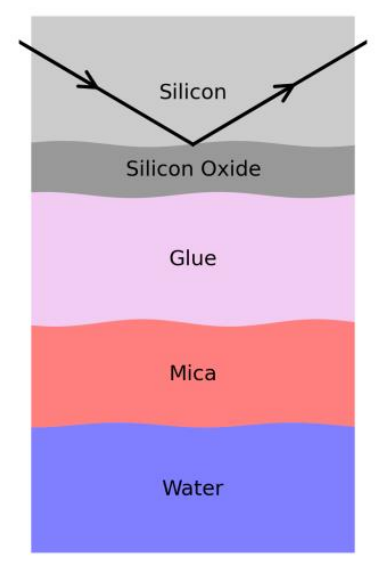

Figure 2: Layered structure of mica supported on a silicon substrate for neutron reflection.

A full description of the mica substrate preparation is detailed elsewhere ${ }^{18}$. In short, a UV-activated glue layer was applied to a silicon substrate, cleaned as above, by spin coating. Meanwhile, a wide adhesive tape was applied to both sides of a muscovite mica sheet (Attwater and Sons) $50 \mathrm{~mm} \times 100 \mathrm{~mm} \times 25 \mu \mathrm{m}$ and the mica carefully cleaved whilst dropping pure water into the newly created surface to facilitate the cleaving process. The adhesive tape-supported mica sheet was carefully applied to the glue coated silicon wafer by swiping a plastic card across the surface. The sample was placed in a plastic clamp, with an optically flat substrate against the flat mica crystal and the glue cured under a UV lamp for 1 hour. Finally the adhesive tape was carefully removed from the mica sheet to reveal a fresh mica surface which was UV Ozone cleaned for 15 minutes to remove any interstitial carbonaceous deposits. The substrate was visually inspected for any bubbling of the mica layer, defects and cracks in the mica or glue and then clamped against a nitric cleaned Teflon trough to make a solid/liquid cell. A schematic of the layered structure of the samples is shown in Figure 2.

\section{Neutron Reflection}


Neutron reflection measurements were made using the SURF reflectometer at the ISIS neutron spallation source, Rutherford Appleton laboratory, Didcot, UK. Reflectivity profiles were measured in time-of-flight mode using three grazing angles of incidence and utilising the full range of neutron wavelengths of the device to cover the required range of momentum transfer. Three angles $\left(\theta=0.25,0.6,1.5^{\circ}\right)$ were used to record data for $\mathrm{Q}$ values between $0.008 \AA^{-1}$ and $0.3 \AA^{-1}$ where $\mathrm{Q}$ is the momentum transfer perpendicular to the surface given by $4 \pi \sin \theta / \lambda$. The footprint of the beam on the sample was $35 \times 70 \mathrm{~mm}$ (solid/liquid interface area in the sample trough was $40 \times 85 \mathrm{~mm}$ ), ensuring that the beam did not clip the edges of the PFTE liquid trough. A series of collimating slits were used to ensure a constant beam footprint at each incident angle; these introduced an instrumental resolution of approximately $4 \%$ at the sample.

Data from reflection measurements has been reduced against a transmission run through a silicon block to account for the beam shape and any attenuation on its passage through the substrate to the detector and a constant background subtracted.

\section{Data Fitting}

The MOTOFIT package has been used to fit the reflectivity data recorded at the silicon/water interface ${ }^{29}$. MOTOFIT calculates the reflectivity arising from a series of thin layers using the Abeles matrix formulism $^{29-31}$. This calculation is valid for the silicon system studied since the layers encountered have thicknesses smaller than the coherence length of the neutron radiation. Here the amplitudes of the reflected beams from the different interfaces in the system should be added ${ }^{31}$. However, to analyse reflection from the mica/water interface an alternative approach must be considered due to the relatively 'thick' mica and glue layers present. 'Thick' layers are those whose thickness is greater than the coherence length of the neutrons radiation. In this case, there is a loss of coherence of the radiation as it passes across the layer from one interface to the other and so reflected intensities, not amplitudes, of emergent beams should be combined ${ }^{18,32}$.

For thin layers, the beams are modulated by a phase term. The intensity of beams from thick layers are modulated by an attenuation term dependent on the total path length through the layer and the wavelength of neutrons (which are not uniformly attenuated by the layer). Neutron reflectivity data recorded from the mica/water interface was fitted using a fitting package (I-CALC) developed in house to include the reflection from thick layers. The I-CALC program applies either thick or thin film calculations to the layers as appropriate as described previously ${ }^{17,18}$.

Reflectivity profiles calculated using the I-CALC program are generated for the three separate angles of incidence and a stitching procedure applied to generate the full profile in exactly the same way as the experimental data. 
The beam attenuation on passage through the thick glue and mica layers has been measured as a function of wavelength. Transmission data is given in the supplementary information. The attenuation cross section is best parameterised as a third order polynomial for the glue layer given in Eq 2.

$$
\begin{aligned}
& N \sigma_{\text {tot,glue }}(\lambda)=\alpha_{\text {glue }}+\beta_{\text {glue }} \lambda+\gamma_{\text {glue }} \lambda^{2}+\delta_{\text {glue }} \lambda^{3} \\
& \alpha_{\text {glue }}=-(0.004 \pm 0.002) \times 10^{-9} \AA^{-1} \\
& \beta_{\text {glue }}=(0.19 \pm 0.07) \times 10^{-9} \AA^{-2} \\
& \gamma_{\text {glue }}=-(5.7 \pm 0.9) \times 10^{-9} \AA^{-3} \\
& \delta_{\text {glue }}=-(31 \pm 4) \times 10^{-9} \AA^{-4}
\end{aligned}
$$

A linear parameterisation is fitted to the wavelength dependent attenuation cross section for the mica data (See Eq 3).

$$
\begin{aligned}
& N \sigma_{\text {tot,mica }}(\lambda)=\alpha_{\text {mica }}+\beta_{\text {mica }} \lambda \\
& \alpha_{\text {mica }}=(-67 \pm 3) \times 10^{-10} \AA^{-1} \\
& \beta_{\text {mica }}=(-4.4 \pm 0.2) \times 10^{-10} \AA^{-2}
\end{aligned}
$$

Instrumental and sample related resolution is included by convolution of the calculated reflectivity profile with full width at half-maximum related to the resolution $\Delta \mathrm{Q} / \mathrm{Q}$. This parameter is fitted as $5 \%$ and $10 \%$ for the silicon and mica substrates respectively.

\section{Results}

\section{Silicon Bare Surface}

To characterise the silica surface, neutron reflectivity profiles were measured in water with three different scattering length densities: $\mathrm{D}_{2} \mathrm{O}, \mathrm{H}_{2} \mathrm{O}$ and water contrast matched to silicon (CMSi). The scattering length densities of each of these components was allowed to float within $0.2 \times 10^{-6} \AA^{-2}$ to allow for any incomplete exchange of liquid in the cells ( $\sim 5 \%$ by volume). Reflectivity profiles are shown in Figure 3 with fits calculated for a $30 \%$ hydrated $12 \AA$ silicon oxide layer with roughness $4 \AA$ at the surface and substrate.

An excellent agreement is found for all these fits, particularly given the highly constrained nature of the structural model using literature values of the material compositions. 


\begin{tabular}{cc}
\hline Material & $\mathbf{S L D} / \times \mathbf{1 0}^{-\mathbf{6}} \mathbf{A}^{-\mathbf{2}}$ \\
\hline Silicon & 2.07 \\
Silicon Oxide & 3.47 \\
Glue & 0.88 \\
Mica & 3.79 \\
$\mathrm{D}_{2} \mathrm{O}$ & 6.3 \\
$\mathrm{H}_{2} \mathrm{O}$ & -0.56 \\
Contrast matched water & 2.05 \\
DDAB & -0.25 \\
\hline
\end{tabular}

Table 2: Scattering length density of materials used during this study.

Scattering length densities for all the materials and solutions used in this study are given in Table 2 . The values for DDAB are based on the bulk density of $0.946 \mathrm{~g} \mathrm{~cm}^{-3}$ as quoted by Grillo et al. in good agreement with values used by Blom et al ${ }^{1,33}$. 

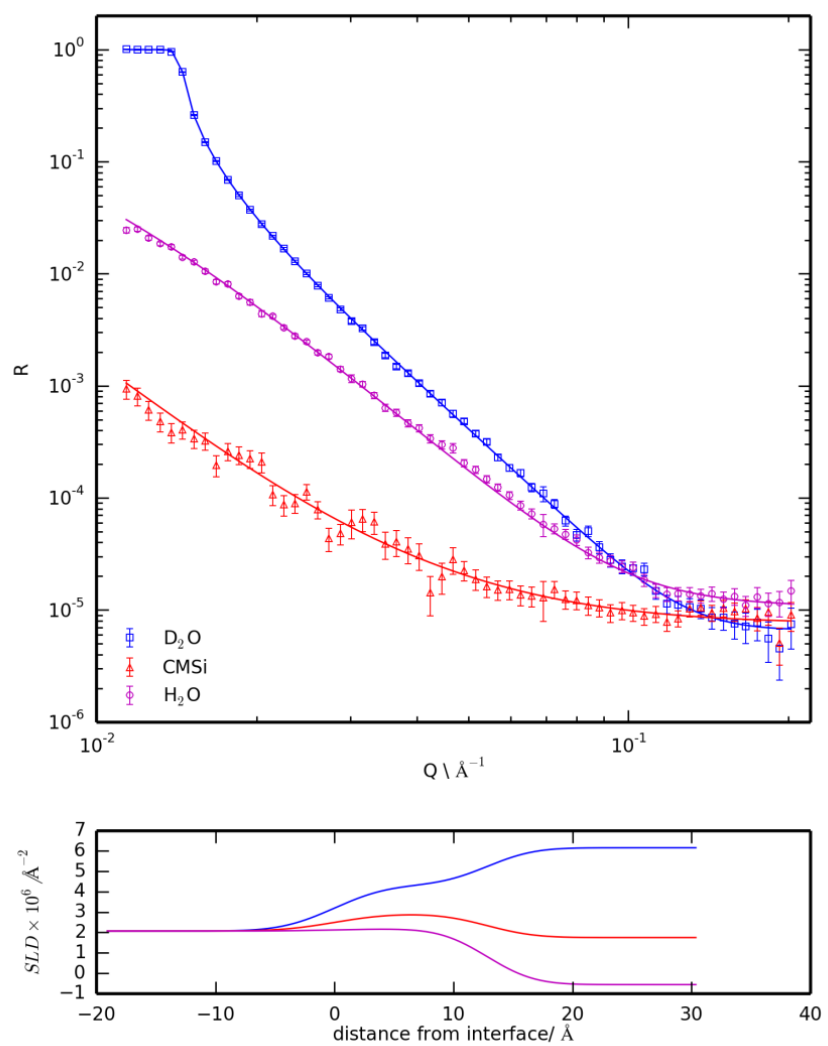

Figure 3: top Neutron reflectivity profiles measured at the silica/water interface plotted on a $\log -\log \operatorname{scale} . \mathrm{D}_{2} \mathrm{O}$ (squares), water contrast matched to silicon (triangles) and $\mathrm{H}_{2} \mathrm{O}$ (circles) contrasts are shown as points whilst continuous lines at fits to the data. bottom SLD profiles for the fits to the data.

\section{DDAB Adsorbed Layer on Silicon}

Figure 4 shows the neutron reflection data for the silica surface with and without adsorbed DDAB at 0.1 $(0.008 \mathrm{mM}), 0.5(0.04 \mathrm{mM})$ and $1 \mathrm{CMC}(0.08 \mathrm{mM})$ in $\mathrm{D}_{2} \mathrm{O}$. Data collected at both 0.5 and $1 \mathrm{CMC}$ show a large change relative to the bare surface unambiguously showing that a surfactant layer has been adsorbed at the silica/water interface. The reflectivity profile for the surface exposed to DDAB at 0.1 CMC shows a much smaller difference from the bare surface. 

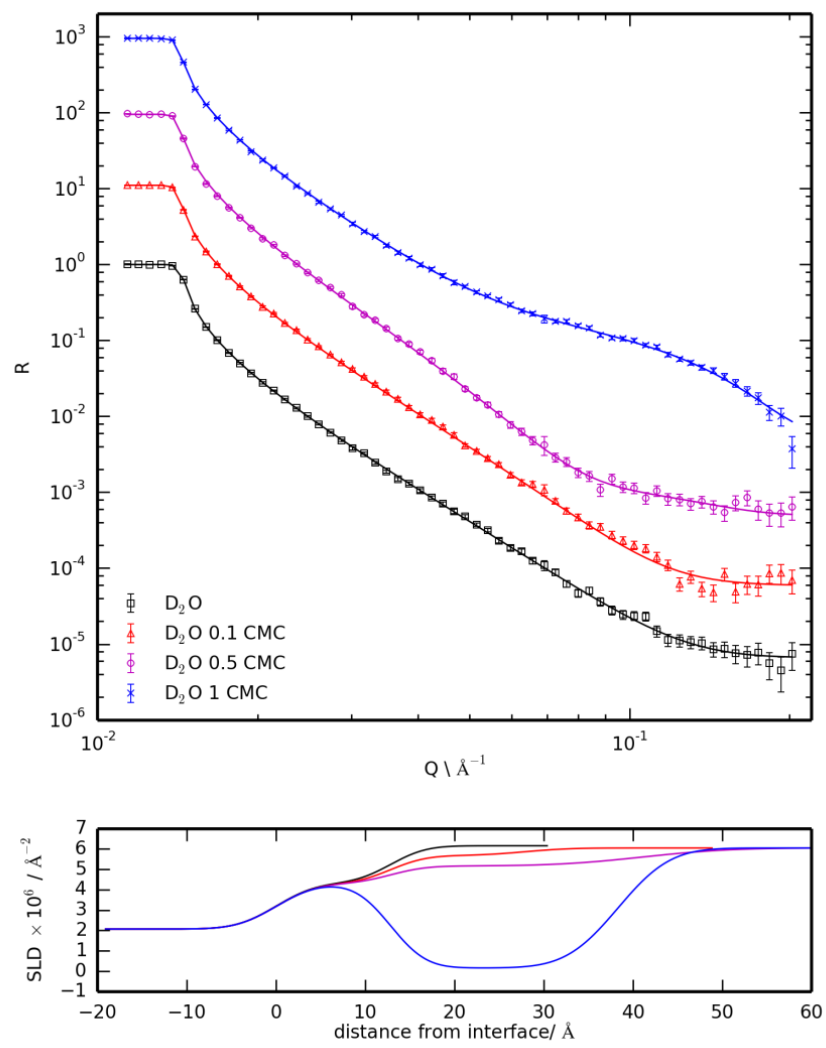

Figure 4: top Neutron reflectivity profiles measured for DDAB adsorbed at the silica/water interface. Data shown is for the surface exposed to $\mathrm{D}_{2} \mathrm{O}$ (squares), 0.1 CMC DDAB (triangles), $0.5 \mathrm{CMC}$ DDAB (circles) and 1 CMC DDAB (crosses). bottom SLD profiles extracted from fits to the data. 0.1 CMC, $0.5 \mathrm{CMC}$ and $1 \mathrm{CMC}$ reflectivity profiles have been offset by factors of ten for clarity.

0.1 CMC and 0.5 CMC DDAB data sets were collected in only one contrast $\left(\mathrm{D}_{2} \mathrm{O}\right)$ and fitted (independently) to a model consisting of a single layer of constant SLD/hydration. The adsorbed layer structure for DDAB at $1 \mathrm{CMC}$ was fully characterised in three contrasts: $\mathrm{D}_{2} \mathrm{O}$ (shown), CMSi and $\mathrm{H}_{2} \mathrm{O}$ (not shown). These three contrasts were fitted simultaneously to a single layer structural model. Only the layer roughness, thickness and solvation were allowed to vary whilst other structural parameters from the substrate were kept constant at the values determined from characterisation of the bare surface. It was found that all the data fitted well to this simple one layer model and so more complex models were not applied.

The data collected for the surfactant adsorbed at $0.1 \mathrm{CMC}$ could be fitted to a wide range of thickness and hydration combinations. This indicates that only a thin or very diffuse surfactant layer is adsorbed at this low concentration. We are confident that some surfactant is adsorbed at the surface because the goodness of fit even to a thin hydrated layer is much better than to just the bare surface. Table 3 shows the parameters used to fit the adsorbed DDAB layers at the three surfactant concentrations studied.

Crucially, the absorbed layer structures are very different at each concentration. At $0.1 \mathrm{CMC}$, very little DDAB is adsorbed. At $0.5 \mathrm{CMC}$, a hydrated bilayer or sparsely adsorbed micelles (14\% DDAB) can be 
interpreted from the fitted parameters and only the CMC is a complete bilayer formed (very little water $7 \%)$.

\begin{tabular}{cccc}
\hline [DDAB]/ CMC & Thickness/ $\AA$ & Hydration/ \% & Roughness/ $\AA$ \\
\hline 0.1 & $15 \pm 9$ & $94 \pm 5$ & $4 \pm 3$ \\
0.5 & $28 \pm 3$ & $86 \pm 2$ & $8 \pm 2$ \\
1 & $25.5 \pm 0.5$ & $7 \pm 1$ & $4.6 \pm 0.3$
\end{tabular}

Table 3: Parameters used to fit the DDAB surfactant layer adsorbed on to the silica surface at $0.1,0.5$ and $1 \mathrm{CMC}$

\section{DDAB Surfactant Removal from Silicon}

The stability of the bilayer formed at the silica/water interface was investigated by washing with (i) pure $\mathrm{D}_{2} \mathrm{O}$, (ii) $10 \mathrm{mM} \mathrm{KCl}$ and (iii) $10 \mathrm{mM} \mathrm{CaCl}_{2}$. The washing procedure entailed pumping the washing solution through the sample cell for $15 \mathrm{mins}$ at a flow rate of $2 \mathrm{~mL} \mathrm{~min}^{-1}$. The reflectivity profile arising from the surface was measured between each stage of the washing process to track the effect on the adsorbed layer. Figure 5 shows the reflectivity profile collected after washing in $\mathrm{D}_{2} \mathrm{O}$ for 15 minutes overlaid on the reflectivity profile of the DDAB layer absorbed at $1 \mathrm{CMC}$. It is clear that the washing procedure had a minimal effect on the surfactant structure. Reflectivity profiles collected after washing for 15 minutes with $10 \mathrm{mM} \mathrm{KCl}$ and after washing for 15 mins with $10 \mathrm{mM} \mathrm{CaCl}_{2}$ are also very similar [given in supplementary information]. The parameters used to fit the adsorbed layer after each stage of washing are given in Table 3. Interestingly, we conclude that these washing procedures have a negligible effect on the adsorbate structure. 


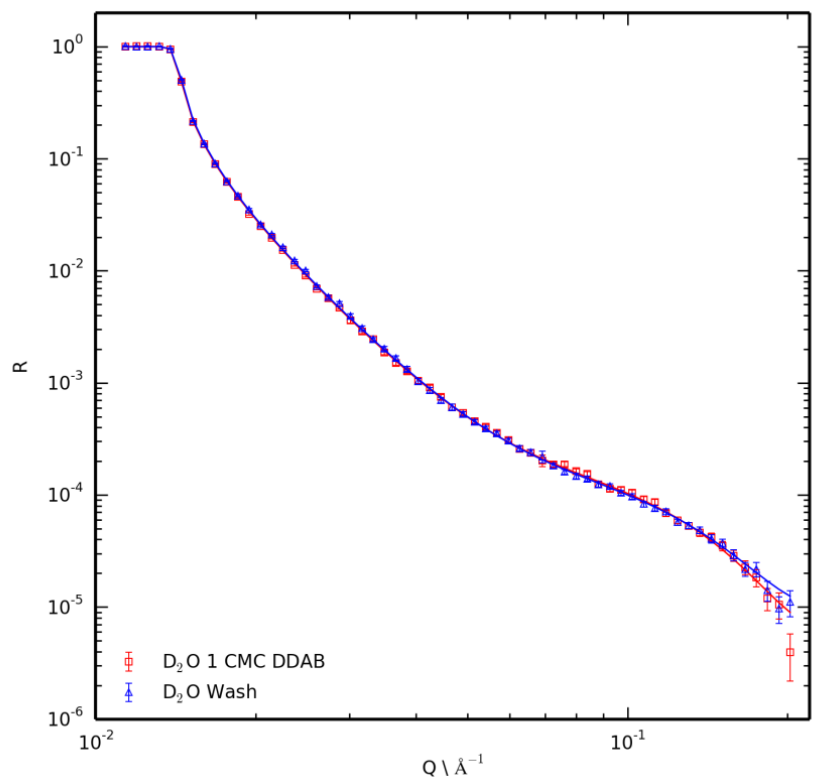

Figure 5: Neutron reflectivity profiles measured at the silica/water interface for the adsorbed bilayer at $1 \mathrm{CMC}^{\mathrm{C}} \mathrm{D}_{2} \mathrm{O}$ (circles) and after washing the surface for 15 minutes in $\mathrm{D}_{2} \mathrm{O}$ (triangles) overlaid showing a small change in the adsorbed layer structure. Points are experimental data, solid lines are fits to the data.

\begin{tabular}{cccc}
\hline Wash & Thickness/ $\AA$ & Hydration/ \% & Roughness/ $\AA$ \\
\hline $\mathrm{D}_{2} \mathrm{O}$ & $25.5 \pm 1.2$ & $8 \pm 3$ & $4.6 \pm 0.7$ \\
$10 \mathrm{mM} \mathrm{KCl}$ & $26.1 \pm 0.3$ & $8.9 \pm 0.5$ & $3.9 \pm 0.4$ \\
$10 \mathrm{mM} \mathrm{CaCl}_{2}$ & $26 \pm 1$ & $8.9 \pm 0.5$ & $6.9 \pm 0.6$
\end{tabular}

Table 4: Parameters used to fit the DDAB surfactant layer remaining at the silica surface after washing in $\mathrm{D}_{2} \mathrm{O}, 10 \mathrm{mM}$ $\mathrm{KCl}$ and $10 \mathrm{mM} \mathrm{CaCl}$. 


\section{Mica Bare Surface}

The bare mica surface was characterised in $\mathrm{D}_{2} \mathrm{O}, \mathrm{CMSi}, \mathrm{H}_{2} \mathrm{O}$, as for silicon above. Fitted reflectivity profiles and SLD plots for the bare mica surface are shown in Figure 6. Here we see a critical edge at a $\mathrm{Q}$ value of $0.009 \AA^{-1}$ in all three data sets corresponding to total reflection from the glue/mica interface. In the $\mathrm{D}_{2} \mathrm{O}$ contrast, two critical edges arising from the silicon/mica and silicon/ $\mathrm{D}_{2} \mathrm{O}$ interfaces are visible at $\mathrm{Q}$ values of $0.009 \AA^{-1}$ and $0.014 \AA^{-1}$ respectively. This confirms that we are able to observe reflection at the mica/ $\mathrm{D}_{2} \mathrm{O}$ interface. The reflected intensity initially falls after the first critical edge but rises again as the second critical edge is approached. This behaviour is a result of the wavelength dependent attenuation of neutrons by the mica and the change in path length through the layer as the refractive index varies with wavelength.
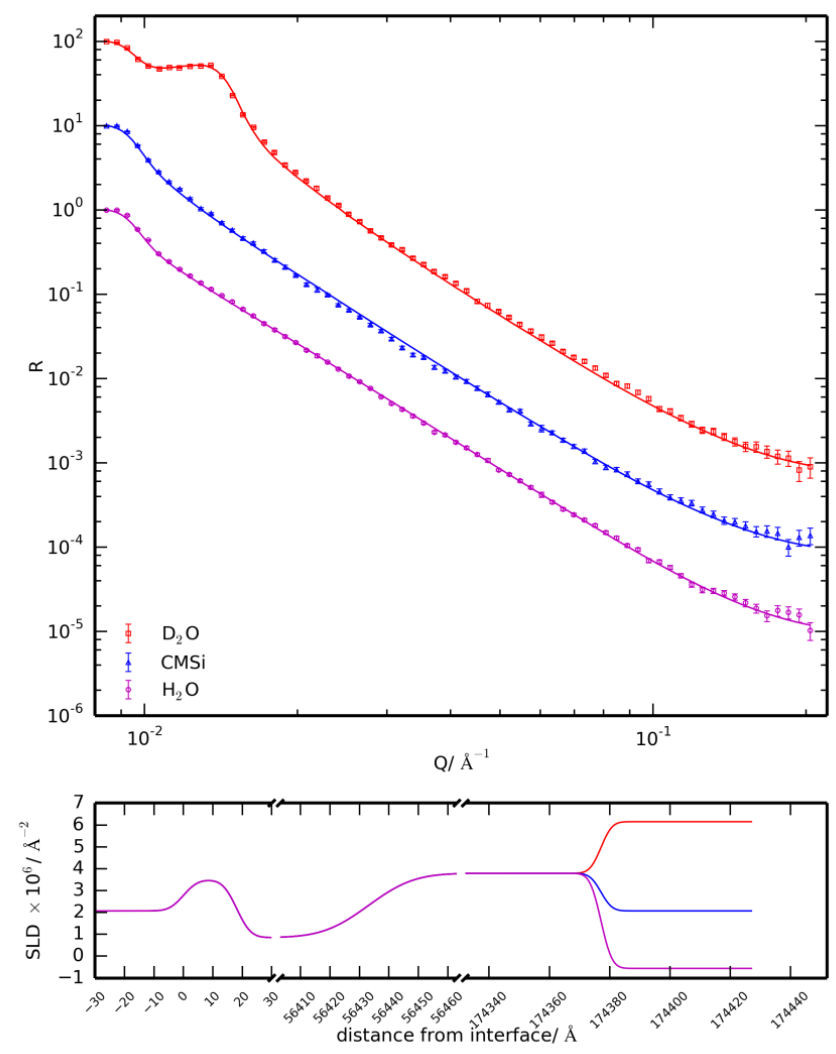

Figure 6: top Neutron reflectivity profiles measured at the mica/water interface plotted on a $\log -\log$ scale. $_{2} \mathrm{O}$ (squares), contrast matched water to silicon (triangles) and $\mathrm{H}_{2} \mathrm{O}$ (circles) experimental data are shown as points. Continuous lines through the data are fits to the data calculated using the parameters given in Table $5 . \mathrm{D}_{2} \mathrm{O}$ and CMSi reflectivity profiles are offset for clarity. bottom SLD profiles extracted from the fits to the data; axes are split so that detail at interfaces of interest is clearly visible 


\begin{tabular}{cccc}
\hline Layer & Thickness & Roughness/ & Hydration/ \% \\
\hline Silicon Oxide & $18 \pm 2 \AA$ & $4 \pm 2$ & 0 \\
Glue & $5.6 \pm 0.4 \mu \mathrm{m}$ & $12 \pm 4$ & 0 \\
Mica & $12 \pm 1 \mu \mathrm{m}$ & $3 \pm 1$ & 0 \\
\hline
\end{tabular}

Table 5: Parameters used for fitting of the bare mica surface reflectivity profiles.

Due to the multilayer substrate, the data in Figure 6 has been fitted to a three layer model corresponding to the native silicon oxide layer of the substrate, the glue layer used to adhere the mica to the substrate and the mica itself, as shown in Figure 2. The parameters used to fit this model are listed in Table 5. The roughness of the substrate is assumed the same as the silicon oxide and a good fit achieved with this assumption. Both the mica and glue layers are 'thick' compared to the coherence length of the neutrons; as discussed above. This procedure has been discussed previously in work addressing the mica/water interface by Browning et al and by Zarbakhsh et al for water and oil layers ${ }^{17,18,32}$.

The fits in Figure 6 are good, given the complex, natural mineral nature of the substrate and the highly constrained nature of the structural model, using literature values of the material compositions.

\section{DDAB Adsorbed Layer on Mica}

Figure 7 shows the reflectivity profiles for the mica surface exposed to DDAB at $0.1,0.5$ and $1 \mathrm{CMC}$ as well as the bare surface for comparison, all recorded in $\mathrm{D}_{2} \mathrm{O}$ (offset for clarity). Fits to the data are shown as solid lines through the data and the extracted SLD profiles also presented.

The measured reflectivity profile after the surface had been exposed to the surfactant at $0.1 \mathrm{CMC}$ is identical to that collected for the bare surface, within experimental error. In Figure 7, the fit to the data is calculated as for the bare surface including no addition layer for the adsorbate at $0.1 \mathrm{CMC}$. Therefore, we conclude that no measureable adsorption has occurred at this concentration, $0.1 \mathrm{CMC}$.

A very clear change in reflectivity is seen for surfactant concentrations of 0.5 and 1 CMC. Qualitatively, it can be seen that the reflectivity and, hence adsorbate structure, is very similar at these two concentrations.

The adsorbed layer at $1 \mathrm{CMC}$ was fully characterised in three contrasts $\left(\mathrm{D}_{2} \mathrm{O}\right.$ [shown], CMSi, $\mathrm{H}_{2} \mathrm{O}$ [see supplementary information]) and these data sets fitted simultaneously to a single structural model. A single layer model was used to fit the data with layer thickness, hydration and roughness allowed to vary. Since a good fit was achieved with this simple model, more complex multi-layered models were not considered. A single layer model for the adsorbate at $0.5 \mathrm{CMC}$ was also employed and fitted to the single $\mathrm{D}_{2} \mathrm{O}$ contrast. 
The parameters used to fit the adsorbate layer at each surfactant concentration are given in Table 6 . We note that the simultaneous fits to these three reflectivity profiles using such a simple highly constrained models is reasonably good, increasing our confidence in the results.

\begin{tabular}{cccc}
\hline $\begin{array}{c}\text { [DDAB }] / \\
\text { CMC }\end{array}$ & Layer Thickness/ $\AA$ & Layer Hydration/ \% & Layer Roughness/ $\AA$ \\
\hline 0.1 & No Adsorption & \\
0.5 & $23 \pm 2$ & $0 \pm 5$ & $2 \pm 1$ \\
1 & $23 \pm 2$ & $0 \pm 5$ & $2 \pm 1$
\end{tabular}

Table 6: Parameters used to fit the DDAB surfactant layer adsorbed on to the mica surface at 0.1, 0.5 and $1 \mathrm{CMC}$
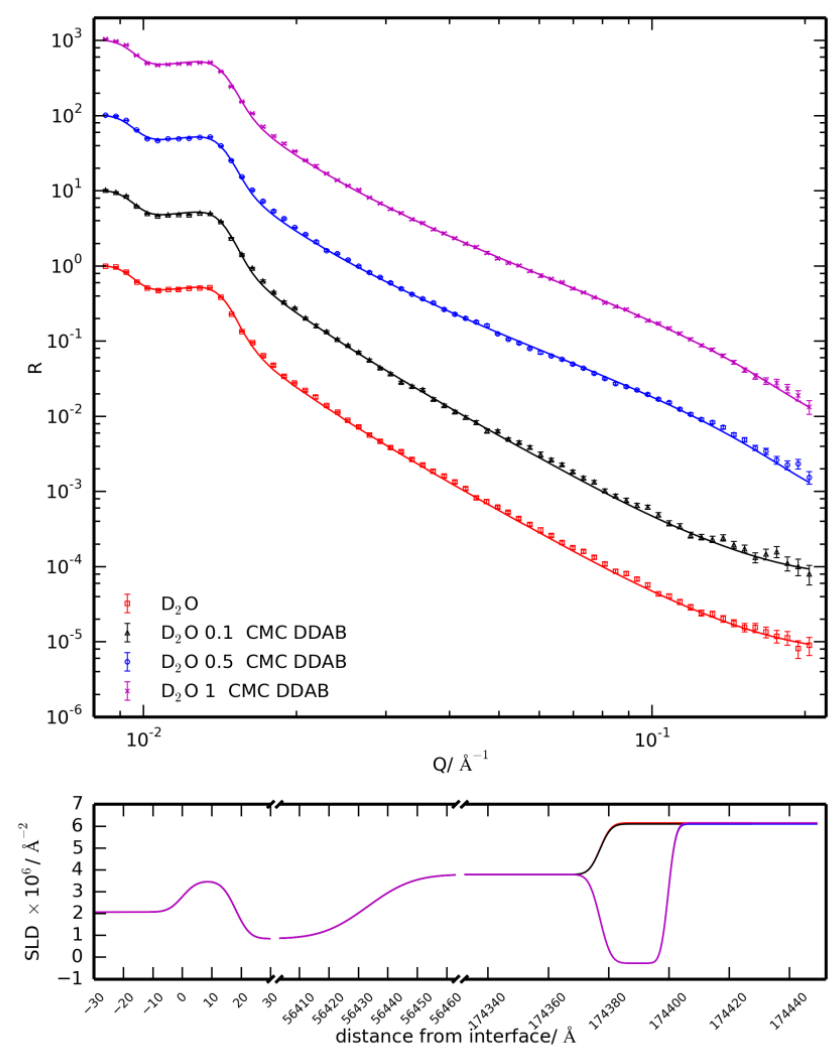

Figure 7: top Neutron reflectivity profiles measured for DDAB adsorbed at the mica/water interface. Data shown is for the surface exposed to $\mathrm{D}_{2} \mathrm{O}$ (squares), $0.1 \mathrm{CMC}$ DDAB (triangles), $0.5 \mathrm{CMC}$ DDAB (circles) and $1 \mathrm{CMC}$ DDAB (crosses). 0.1 CMC, 0.5 CMC and 1 CMC reflectivity profiles have been offset for clarity. bottom SLD profiles for the fits to the data.

\section{DDAB Surfactant Removal from Mica}


Bilayer stability at the mica/water interface was investigated by washing with (i) $\mathrm{D}_{2} \mathrm{O}$, (ii) $10 \mathrm{mM} \mathrm{KCl}$ and (iii) $10 \mathrm{mM} \mathrm{CaCl}_{2}$ by passing the solution through the sample cell at $2 \mathrm{~mL} \mathrm{~min}^{-1}$ for 15 minutes. The reflectivity profile was measured between each stage of the washing process to track the effect on the adsorbed layer. Figure 8 shows the reflectivity profile collected after washing in $\mathrm{D}_{2} \mathrm{O}$ for 15 minutes overlaid on the reflectivity profile of the DDAB layer absorbed at $1 \mathrm{CMC}$ (data collected after washing in $10 \mathrm{mM} \mathrm{KCl}$ and with $10 \mathrm{mM} \mathrm{CaCl}_{2}$ are very similar to the $\mathrm{D}_{2} \mathrm{O}$ wash [see supplementary information]).

The adsorbed layer remaining after each stage of washing was fitted to a single layer model allowing the layer thickness, roughness and hydration to vary. Parameters extracted from the fitting procedure are given in Table 7.

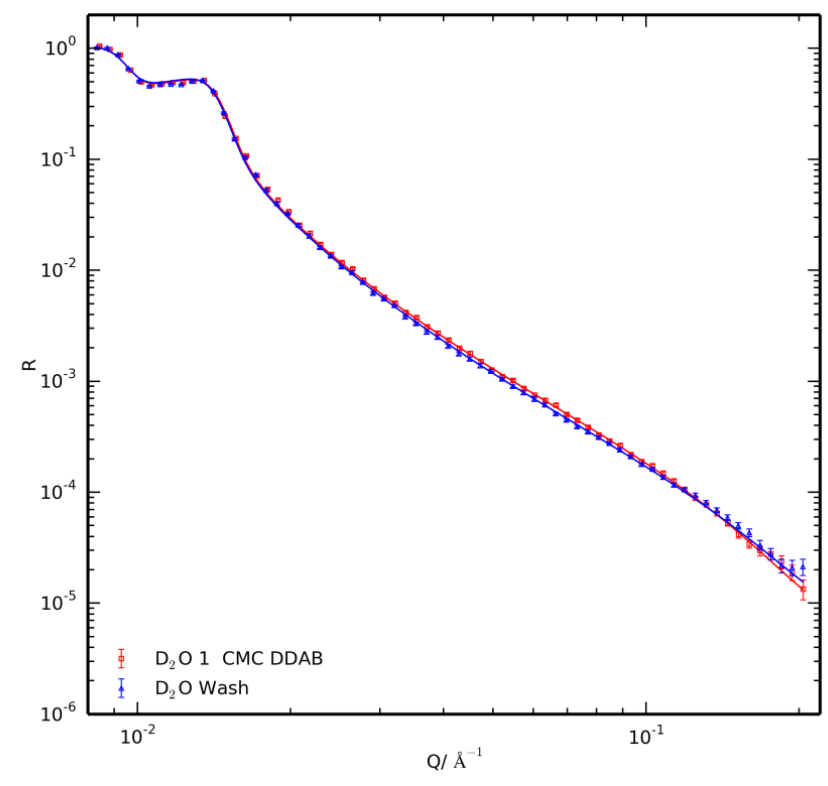

Figure 8: Neutron reflectivity profiles measured at the mica/water interface for the adsorbed bilayer at $1 \mathrm{CMC}^{\text {in }} \mathrm{D}_{2} \mathrm{O}$ (squares) and after washing the surface for 15 minutes in $\mathrm{D}_{2} \mathrm{O}$ (diamonds) are overlaid, showing a small change in the adsorbed layer structure. Data are plotted on a log-log scale.

\begin{tabular}{cccc}
\hline Wash & $\begin{array}{c}\text { Layer Thickness/ } \\
\AA\end{array}$ & $\begin{array}{c}\text { Layer } \\
\text { Hydration/ \% }\end{array}$ & $\begin{array}{c}\text { Layer Roughness/ } \\
\AA\end{array}$ \\
\hline $\mathrm{D}_{2} \mathrm{O}$ & $20 \pm 1$ & $0 \pm 2$ & $2 \pm 1$ \\
$10 \mathrm{mM} \mathrm{KCl}$ & $20 \pm 1$ & $0 \pm 2$ & $2 \pm 1$ \\
$10 \mathrm{mM} \mathrm{CaCl}_{2}$ & $20 \pm 1$ & $0 \pm 2$ & $2 \pm 1$
\end{tabular}

Table 7: Parameters used to fit the DDAB surfactant layer remaining at the silica surface after washing in $\mathrm{D}_{2} \mathrm{O}, 10 \mathrm{mM}$ $\mathrm{KCl}$ and $10 \mathrm{mM} \mathrm{CaCl}$. 


\section{Discussion}

Charge density of the substrate has been shown to affect the final adsorbed layer structure for a given surfactant ${ }^{13}$. Adsorption of cationic surfactants on oxide surfaces (like silica) has been reasonably well documented and the isotherm is thought to exhibit three stages driven by a balance of electrostatic and hydrophobic forces, as now outlined. First, individual surfactants molecules are adsorbed electrostatically to the charged surface. The adsorption of surfactant headgroups at the surface makes the hydroxyl groups close by more acidic thus allowing the formations of more charged sites. Second, the hydrophobic tails of the existing surfactants on the surface along with the newly created charged sites encourages adsorption of more surfactant molecules surrounding those already adsorbed. In effect, the initially adsorbed surfactants act as nucleation points for further adsorption. At the end of this stage, the overall surface charge is neutralised. In the third stage, hydrophobic interactions drive the adsorption. Further surfactant molecules cluster around those already adsorbed at the surface from the second stage. These have their headgroups directed away from the surface completing an aggregate structure at the surface ${ }^{26}$.

\section{Silica}

It can be seen from Table 3 that the DDAB adsorbate layer becomes thicker with increasing surfactant concentration whilst the layer hydration and roughness decrease. These observations are consistent with an increased surface coverage as the surfactant concentration in free solution increases. The adsorbed layer measured at $1 \mathrm{CMC}$ is consistent with a bilayer, as observed on quartz ${ }^{1,6}$. On silica the layer is $7 \%$ hydrated by volume whereas on quartz the layer is $6 \%$ hydrated.

The measured bilayer thickness is less than twice the extended chain length of the DDAB surfactant, as calculated by Tanford's formula, suggesting interdigitation of the surfactant chains or a tilting of the bilayer by approximately $40^{\circ}$ to the surface normal ${ }^{34}$.

Measurements of the adsorbed layer below the CMC suggest it is appreciably thinner than the bilayer adsorbed at the CMC. At 0.1 CMC, a $94 \%$ hydrated $15 \AA$ layer is adsorbed; this fit to the data is not necessarily unique but does show that minimal surfactant is absorbed at this low concentration.

Upon increasing the surfactant concentration to $0.5 \mathrm{CMC}$, the layer thickens to $28 \AA$ and its hydration is reduced to $86 \%$. We suggest the formation of a patchy bilayer at the surface where surfactant molecules in the layer orientated closer to the surface normal than in the bilayer $\left(33^{\circ}\right.$ c.f. $\left.40^{\circ}\right)$. More surfactant is adsorbed at this higher concentration but full surface coverage has not been reached.

Partial surfactant coverage of the surface at concentrations below the CMC is reported by Atkin et al for $\mathrm{CTAB}$ on silica ${ }^{35}$. Their ellipsometric measurements indicate that below a critical concentration (named the Critical Surface Aggregation Concentration, CSAC) a fractional layer is adsorbed and only at concentrations above the CSAC is a full layer adsorbed ( $0.6 \mathrm{CMC}$ for the CTAB). This surfactant assembly behaviour mirrors our findings for DDAB on silica below the CMC. We note however, that 
literature makes it clear that the preparation of the silica/silicon surface can significantly affect its behaviour $^{35,36}$. Our silicon preparation procedure is given above (see Materials and Methods section).

\section{Mica}

Table 6 shows the parameters which characterise the DDAB adsorbed layer at 0.1, 0.5 and $1 \mathrm{CMC}$. By contrast to silicon/silica no adsorbed layer is measured at 0.1 CMC. A $23 \AA$ unhydrated layer is measured at both $0.5 \mathrm{CMC}$ and $1 \mathrm{CMC}$, interpreted as an essentially complete bilayer structure.

Formation of a uniform unhydrated bilayer below the DDAB CMC (first observed at $0.5 \mathrm{CMC}$ ) is an interesting and unexpected result. Completion of the final aggregate structure as low as $0.5 \mathrm{CMC}$ on mica differs from the view that adsorption to hydrophilic surfaces switches on sharply near the $\mathrm{CMC}^{37}$.

\section{Comparison}

The neutron reflection measurements given here show that the identity of the surface affects the adsorption behaviour of the surfactant, DDAB. At $0.5 \mathrm{CMC}$, the free solution concentration of DDAB is not sufficient to fully adsorb on the silica surface whereas assmebly at the mica surface is essentially complete. The differing nature of the adsorption of these two surfaces conflicts with the traditional view that the silica and mica surfaces can be treated as similar and somewhat interchangeable.

Possible explanations for these differences observed in the adsorption isotherms for DDAB on the mica and silica surfaces include the difference in surface charge and flatness of the surface.

In the traditional model for surfactant adsorption at charged surfaces, it is suggested that the initial adsorption step is driven by electrostatic attraction. Adsorption remains low until the CSAC where the surface excess rapidly increases due to cooperative adsorption is reached (typically 0.6 - 0.9 CMC). Adsorption typically plateaus by the $\mathrm{CMC}^{26,37}$. The charge density of the mica surface is much greater than for silica. We suggest that the higher charge of the mica drives the surfactant adsorption to occur at a lower bulk concentration, resulting in complete bilayer adsorption at a lower bulk concentration than the lower charge silica.

Cleaving mica along the basal plane achieves large step free areas $\left(>1 \mathrm{~cm}^{2}\right.$ for high quality mica $\left.{ }^{38}\right)$, the local atomic flatness achieved in this cleaving process is not necessarily achieved during the silicon substrate polishing procedures. Fragneto et al found that the $\mathrm{C}_{16} \mathrm{TAB}$ adsorbate structure and the surface coverage on silica was affected by substrate roughness. On rough substrates a lower surface coverage was achieved than for smooth substrates ${ }^{5}$. It is possible that the flatness of the mica substrate enhances favourable interactions between adsorbed surfactant molecules in a way that encourages full bilayer formation at lower free solution concentrations than for silica.

\section{Surfactant Removal}


The DDAB surfactant on both silica and mica surfaces proved rather tenacious. Attempted removal by washing with $\mathrm{D}_{2} \mathrm{O}, 10 \mathrm{mM} \mathrm{KCl}$ and then $10 \mathrm{mM} \mathrm{CaCl}_{2}$ had minimal effect on the layer. We conclude that ion exchange as a method for removal of the DDAB surfactant from silica or mica layers was ineffectual and the bilayer remained essentially complete. It is suggested that hydrophobic interactions within the complete layer contribute to the irreversibility of the adsorption.

\section{Conclusions}

We have applied neutron reflection to the study of DDAB on both silica and mica surfaces, providing a direct comparison on the adsorption on the two surfaces. The adsorption behaviour of this surfactant was studied at a range of concentrations up to and including the CMC. Key findings include the formation of a complete bilayer at the $\mathrm{CMC}$ for both the mica and silicon surfaces whilst the adsorbed layer structure showed differences between these two substrates at concentrations below the CMC. The thickness of the bilayer adsorbed at the CMC determined from our measurements was in good agreement with the previous measurements of DDAB on silica by Schultz et al and Blom et al and on mica by Browning et $a l^{1,6,18}$. Most notably a complete bilayer was adsorbed at the mica/water interface at just half the CMC whilst still incomplete at this concentration on silica. Hence our hypothesis is that the mica and silica adsorb these additives rather differently. In some senses this is not surprising given that mica has a high structural charge density and silica has no structural charge but only a $\mathrm{pH}$ dependent surface charge. To the author's best knowledge this is the first reported difference in DDAB adsorption on mica and silica surfaces below the CMC. The high mica charge or surface smoothness are suggested as possible explanations for this difference.

The adsorbed layer was found to be particularly tenacious on the surface. Removal by washing with water or ion exchange with $\mathrm{K}^{+}$or $\mathrm{Ca}^{2+}$ had a negligible effect on the adsorbed layer.

These findings demonstrate that DDAB surfactant assembly behaviour is dependent on the identity of the surface itself below the CMC. This research highlights that caution should be exercised when comparing adsorbate behaviour on mica (a common substrate in AFM and SFA studies) and silica (routinely used for neutron reflection measurements).

We have already some preliminary data from other anionic organics that show much more dramatic differences in adsorption between mica and silica, that we intend to complete in the near future along with related comparisions on mica and silica of polymeric species which are very important commercially. 


\section{Acknowledgements}

We would like to thank the beam time committee for awarding the time to undertake this study at ISIS (RB 1320079 and RB 1320050) along with the beamline scientists for their help and guidance during the experiments and data analysis. Further thanks are extended to Andy Pluck (BP Institute), Andy Church (ISIS Sample environment), Alasdair Ross and Sean Peacock (University of Cambridge Engineering Department) for help manufacturing and designing sample cells. We thank BP for funding the work and our industrial colleagues, Isabella Stocker and Peter Salino, for their thoughts and contributions to the work (RG63491). Finally, we thank Robert Thomas and Giovanna Fragneto for their valuable contributions and discussions during preparation and revision of the manuscript. 


\section{References}

(1) Blom, A.; Warr, G. G.; Nelson, A. Colloids Surfaces A Physicochem. Eng. Asp. 2007, 310 (1-3), $1-8$.

(2) Penfold, J.; Thomas, R. K. J. Phys. Condens. Matter 1990, 1369-1412.

(3) Li, N.; Thomas, R. K.; Rennie, A. R. J. Colloid Interface Sci. 2012, 369 (1), 287-293.

(4) Rennie, A. R.; Lee, E. M.; Simister, E. A.; Thomas, R. K. Langmuir 1990, 6 (5), 1031-1034.

(5) Fragneto, G.; Thomas, R. K.; Rennie, A. R.; Penfold, J. Langmuir 1996, 12 (25), 6036-6043.

(6) Schulz, J.; Warr, G.; Butler, P.; Hamilton, W. A. Phys. Rev. E 2001, 63 (4), 041604-1 - 041604041605 .

(7) McDermott, D. C.; Kanelleas, D.; Thomas, R. K.; Rennie, a. R.; Satija, S. K.; Majkrzak, C. F. Langmuir 1993, 9 (9), 2404-2407.

(8) McDermott, D. C.; McCarny, J.; Thomas, R. K.; Rennie, A. R. J. Colloid Interface Sci. 1994, 162, 304-310.

(9) McDermott, D. C.; Lu, J. R.; Lee, E. M.; Thomas, R. K.; Rennie, a. R. Langmuir 1992, 8 (4), 1204-1210.

(10) Fujita, M.; Takikawa, Y.; Sakuma, H.; Teramachi, S.; Kikkawa, Y.; Doi, Y. Macromol. Chem. Phys. 2007, 208 (17), 1862-1870.

(11) Araujo, P. T.; Barbosa Neto, N. M.; Chacham, H.; Carara, S. S.; Soares, J. S.; Souza, a D.; Cançado, L. G.; de Oliveira, a B.; Batista, R. J. C.; Joselevich, E.; Dresselhaus, M. S.; Jorio, a. Nano Lett. 2012, 12 (8), 4110-4116.

(12) Cosgrove, T.; Heath, T. G.; Phipps, J. S.; Richardson, R. M. Macromolecules 1991, 24, 94-98.

(13) Manne, S.; Gaubt, H. E. Science (80-. ). 1995, 270 (5241), 1480-1482.

(14) Dante, S.; Hauss, T.; Steitz, R.; Canale, C.; Dencher, N. A. Biochim. Biophys. Acta 2011, 1808 (11), 2646-2655.

(15) Su, T.; Lu, J.; Thomas, R.; Cui, Z.; Penfold, J. J. Colloid Interface Sci. 1998, 203 (2), 419-429.

(16) Van Olphen, H. In An Introduction to Clay Colloidal Chemistry; 1977.

(17) Griffin, L. R.; Browning, K. L.; Truscott, C. L.; Clifton, L. A.; Clarke, S. M. J. Phys. Chem. B 2015, 119, 6457-6461.

(18) Browning, K. L.; Griffin, L. R.; Gutfreund, P.; Barker, R. D.; Clifton, L. A.; Hughes, A.; Clarke, S. M. J. Appl. Crystallogr. 2014, 47, 1638-1646.

(19) Ralph K. Iler. The Chemistry of Silica; John Wiley \& Sons, 1979.

(20) Schlegel, M. L.; Nagy, K. L.; Fenter, P.; Cheng, L.; Sturchio, N. C.; Jacobsen, S. D. Geochim. Cosmochim. Acta 2006, 70 (14), 3549-3565.

(21) Olphen, V. Introduction to Clay Colloidal Chemistry, 2nd ed.; Wiley, 1977.

(22) Nishimura, S.; Scales, P. J.; Healy, T. W.; Tsunematsu, K.; Tateyamat, T. 1994, No. 17, 45544559 .

(23) Pashley, R. .; Israelachvili, J. . Journal of Colloid and Interface Science. 1984, pp 446-455.

(24) Evans, D. F.; Wennerstrom, H. In The Colloidal Domain: Second Edition; 1999; pp 99-152.

(25) Dubois, M.; Zemb, T. 1991, No. 11, 1352-1360.

(26) Atkin, R.; Craig, V. S. J.; Wanless, E. J.; Biggs, S. Adv. Colloid Interface Sci. 2003, 103 (3), 219- 
304.

(27) Para, G.; Hamerska-Dudra, A.; Wilk, K. A.; Warszyński, P. Colloids Surfaces A Physicochem. Eng. Asp. 2011, 383 (1-3), 67-72.

(28) Soltero, J. F. a.; Bautista, F.; Pecina, E.; Puig, J. E.; Manero, O.; Proverbio, Z.; Schulz, P. C. Colloid Polym. Sci. 2000, 278 (1), 37-47.

(29) Nelson, A. J. Appl. Crystallogr. 2006, 39 (2), 273-276.

(30) Nelson, A. J. Phys. Conf. Ser. 2010, 251, 012094.

(31) Heavens, O. S. Optical Properties of Thin Solid Films; Dover Publications, 1955.

(32) Zarbakhsh, A.; Querol, A.; Bowers, J.; Webster, J. R. P. Faraday Discuss. 2005, 129, 155-167.

(33) Grillo, I.; Penfold, J.; Tucker, I.; Cousin, F. Langmuir 2009, 25 (7), 3932-3943.

(34) Tanford, C. J. Phys. Chem. 1972, 76 (21), 3020-3024.

(35) Atkin, R.; Craig, V. S. J.; Biggs, S. Langmuir 2000, 16 (8), 9374-9380.

(36) Penfold, J.; Staples, E.; Tucker, I. 2002, No. 12, 2967-2970.

(37) Tiberg, F.; Joensson, B.; Tang, J.; Lindman, B. Langmuir 1994, 10 (7), 2294-2300.

(38) de Poel, W.; Pintea, S.; Drnec, J.; Carla, F.; Felici, R.; Mulder, P.; Elemans, J. a. a. W.; van Enckevort, W. J. P.; Rowan, A. E.; Vlieg, E. Surf. Sci. 2014, 619, 19-24. 